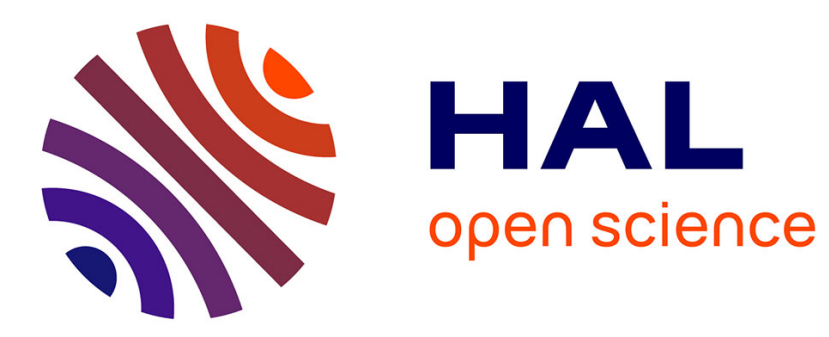

\title{
Glasses as ultrasound detectors
}

\author{
P. Doussineau, A. Levelut, Ta Thu-Thuy
}

\section{To cite this version:}

P. Doussineau, A. Levelut, Ta Thu-Thuy. Glasses as ultrasound detectors. Journal de Physique Lettres, 1978, 39 (4), pp.55-57. 10.1051/jphyslet:0197800390405500 . jpa-00231441

\section{HAL Id: jpa-00231441 https://hal.science/jpa-00231441}

Submitted on 1 Jan 1978

HAL is a multi-disciplinary open access archive for the deposit and dissemination of scientific research documents, whether they are published or not. The documents may come from teaching and research institutions in France or abroad, or from public or private research centers.
L'archive ouverte pluridisciplinaire HAL, est destinée au dépôt et à la diffusion de documents scientifiques de niveau recherche, publiés ou non, émanant des établissements d'enseignement et de recherche français ou étrangers, des laboratoires publics ou privés. 


\title{
GLASSES AS ULTRASOUND DETECTORS
}

\author{
P. DOUSSINEAU, A. LEVELUT and TA THU-THUY
}

Laboratoire d'Ultrasons $\left({ }^{*}\right)$, Université Pierre-et-Marie-Curie, Tour 13, 4, place Jussieu, 75230 Paris Cedex 05, France

(Reçu le 15 novembre 1977, révisé le 23 décembre 1977, accepté le 4 janvier 1978)

\begin{abstract}
Résumé. - Les propriétés élasto-électriques d'un verre contenant des impuretés polaires sont utilisées pour la détection en volume d'ondes ultrasonores. Les avantages et les limites de cette méthode sont comparés à ceux de méthodes plus usuelles : piézoélectricité, détection bolométrique.

Abstract. - The elastoelectric properties of a glass with polar impurities are used for phaseinsensitive detection of ultrasonic waves. The advantages and limitations of this method are compared to those of the more commonly used methods using piezoelectric, or bolometric detection.
\end{abstract}

1. Introduction. - Ultrasonic waves are a well known probe for studying various phenomena in nature, particularly in physics. From attenuation and phase velocity measurements of high frequency sound waves, one can observe interactions between phonons (having a well-defined frequency, wave vector and polarization) and various different excitations in solids such as electrons, spins, magnons and other phonons [1]. The first obstacle the experimentalist must overcome correctly is the generation and above all the detection of elastic waves.

Coherent elastic waves are often generated by electromagnetic excitation of a piezoelectric material. In the high frequency range it has been shown that ultrasonic waves are excited by gradients of an alternating piezoelectric stress, for instance at a free surface [2]. This is of prime importance to obtain a phase reference for the ultrasonic generation. When detected (by the inverse process), the different elementary ultrasonic amplitudes are integrated over the whole sample end-face. In principle a correct signal is obtained if the wave surface and the end-face are perfect parallel planes.

If we consider an idealized propagation of an ultrasonic wave train in a homogeneous medium, the detected image as seen on an oscilloscope would consist of a set of equidistant echoes, decreasing exponentially due to the losses which are related to the successive reflections of the trains in the sample. Then it is straightforward to deduce the attenuation coefficient. In practice it is not so simple and the detected image is often perturbed for example by crystal inhomogeneities, lack of parallelısm or planity

(*) Associated with the Centre National de la Recherche Scientifique. of the free faces... The detected echoes present an important though apparent level loss, due to the phase average on the receiving face.

Various methods have been proposed to cope with these problems : superconducting bolometer [3], phonon echoes [4], Brillouin diffraction [5], semiconducting avalanche bolometer [6], and Josephson junctions [7]. We present here a new way of solving the problem of the detection of coherent elastic waves using some new properties characteristic of amorphous insulators (glasses) at low temperatures.

2. Glass properties. - Many thermal and acoustic experiments [8] have given evidence for the existence of specific low energy excitations in amorphous materials. It has also been shown that these twolevel systems (T.L.S.) are strongly coupled with strains. More recently, experiments mixing acoustic and electromagnetic waves inside an amorphous material containing polar impurities, revealed that the same T.L.S. could be strongly coupled with both waves $[9,10]$. Consequently these systems can be used as good detectors in the following way : the ultrasonic excitation of the T.L.S. changes the electric impedance of a resonant cavity in which the glass sample is introduced; the spectrometer balance is then destroyed and an electromagnetic signal appears [9]. In this energy transformation the glass is active over its volume, and only the acoustic intensity not the phase, is important, because the quantity finally observed is a change of the T.L.S. population. When using this method of sound detection. we must pay attention to amorphous specificities. Particularly the T.L.S. may have a non-linear behaviour as, because of their finite number, they can be saturated at high ultrasonic power levels [8]. 
3. Experimental method. - To demonstrate the validity of this detection process, we bound a long (piezoelectric) X-cut crystal quartz rod onto a borosilicate (BK 7) sample. The amorphous properties of BK 7 are quite well known [8]. The quartz rod is introduced in a reentrant cavity (No1) which produces and detects ultrasonic waves by piezoelectricity. The thin BK 7 sample $(1 \mathrm{~mm})$ is introduced into a similar cavity (No 2), whose impedance depends on the complex dielectric constant $\varepsilon(v)$ of the glass; the interaction of the T.L.S. with the ultrasonic wave at frequency $v_{\text {us }}$ modifies $\varepsilon(v)$ for $v \simeq v_{\text {us }}$. The impedance variation of cavity 2 is detected via a reflection spectrometer. The frequency $v$ is near $9 \mathrm{GHz}$ in our experiment. The endface of the BK 7 glass is ground to avoid spurious reflections. Moreover the bond is not to good in order not to perturb the quartz attenuation : the loss by transmission in BK 7 must be low.

Through cavity 1 , we obtain the echoes sketched in figure 1 by the bars. Their decay envelope is very irregular and inexploitable. Through cavity 2, we observe echoes due to the unbalance of the spectrometer by the arrival of ultrasonic pulses in the glass sample. In order to avoid the difficulties which arise from the non-linear properties of the glass, we use the following procedure. The electromagnetic power level in the spectrometer which contains cavity 2 is kept constant at a value of about $10 \mu \mathrm{W}$. (For comparison, the mean electromagnetic power is two orders of magnitude higher in cavity 1 where ultrasonic waves are generated by direct piezoelectric conversion). We take a far echo seen through cavity 2 as an intensity reference (for the case of figure $1: 14$ th echo). Then, with a calibrated attenuator which diminishes the power entering cavity 1 , we adjust the level of a near echo observed in cavity 2 to be equal to the reference level and we read the number of $\mathrm{dB}$ on the

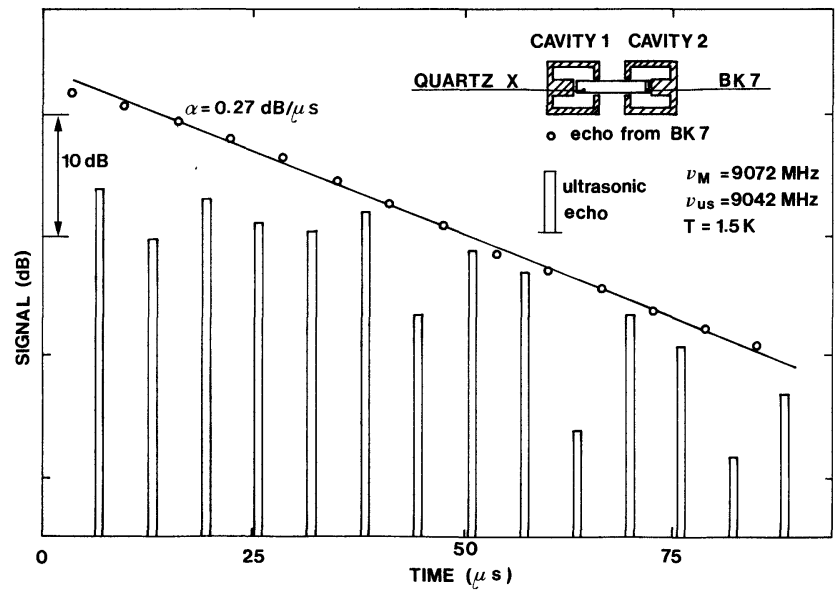

FIG. 1. - The insert represents the experimental set-up : cavity 1 is used to generate and detect the ultrasonic pulses both by piezoelectricity; cavity 2 which contains the glass sample is a part of a microwave reflection spectrometer. In the figure the bars represent the intensity (measured in $\mathrm{dB}$ ) of the ultrasonic pulses detected by piezoelectric surface excitation in cavity 1 . The open circles give the decay of the pulses measured by volume detection via the glass sample in cavity 2 . attenuator. When the procedure is applied successively to each of the echoes we obtain the set of open circles in figure 1. The points lie on a straight line, the slope giving the attenuation coefficient in $\mathrm{dB}$ per $\mu \mathrm{s}$. With this procedure, we have only assumed that equal echo levels are given by equal acoustic fluxes entering the BK 7 sample. Another assumption is that there is no cumulative effect in the $\mathrm{BK} 7$ sample due to the successive echoes. In other words we have supposed that the relaxation tıme $T_{1}$ of the T.L.S. population is shorter than the delay $\tau=2 l ; v$ for a round trip in the quartz crystal ; this condition is quite well satisfied as $\tau \simeq 6 \mu$ s and $T_{1} \simeq 0.1$ or $1 \mu \mathrm{s}$ in our temperature and frequency ranges [11]. The two cavities are not tuned to the same frequency. The difference is $\Delta v=30 \mathrm{MHz}$ which is larger than the cavity bandwidths but smaller than the T.L.S. bandwidth; therefore the ultrasonic wave is not detected directly in cavity 2 by the piezoelectric effect on the quartz face but its effect on T.L.S. is still sensitive because it saturates the population on a broad bandwidth [11]. We have also verify that the attenuation coefficient measured in the quartz crystal is independent of the microwave power in cavity 2 .

4. Comments on the method. - This new detection method of ultrasonic pulses is carried out over the volume and therefore is insensitive to phase effects; this is the main property. As well as underlining some other advantages, we mention some limitations and compare it to other methods of detection. Our method can be used even in a strong magnetic field because the properties of the T.L.S. are field independent.

It can be applied in a large frequency range because the T.L.S. spectrum is very broad (typically from 0 to $0.1 \mathrm{eV}$ ); but some limitations occur because of the relaxation time $T_{1}$ which must be short. The other main limitations come from the T.L.S. population which must be far from saturation in order that the sensitivity be sufficient : this requires a low temperature (in practice $T<5 \mathrm{~K}$ ) and an ultrasonic power level neither too high (less than $10^{-3} \mathrm{~W} . \mathrm{cm}^{-2}$ in order to avoid saturation) nor too low (larger than $10^{-8} \mathrm{~W} . \mathrm{cm}^{-2}$ for having a measurable signal). The sensitivity of our method is good because it avoids piezoelectric detection. whose efficiency is typically $10^{-4}$ at $10 \mathrm{GHz}$. With a good bond, the gain of detection sensitivity is estimated to be 10 or $20 \mathrm{~dB}$ at $1.5 \mathrm{~K}$ and $10 \mathrm{GHz}$.

In fact the sensivity is directly related to the magnitude of the resonant attenuation of ultrasonic waves by T.L.S. This attenuation varies as $\omega^{2} T^{-1}$, therefore the sensivity of our detector decreases as $T^{-1}$ when $T$ increases. Another limitation comes from the non-resonant part of the ultrasound attenuation which varies as $T^{3}$. But its order of magnitude does not rule out the possibility of the glass detector working even at $4.2 \mathrm{~K}$. We have in fact tested that our detector is operative at this temperature. 
As a function of the power level entering the detector cavity, the sensitivity increases linearly with power as long as its magnitude is lower than a critical value above which the T.L.S. population is partially saturated. We have operated with an electromagnetic power in cavity 2 lower than the critical value $(50 \mu \mathrm{W}[9])$.

The question of magnetic field introduces a difference with the same method applied to true spins : a biasing magnetic field is necessary to match the spin energy splitting to phonon energy and then $H$ is no longer a free parameter.

Superconducting bolometers where a flux of acoustic energy induces a change of temperature have not proved too useful to date as their sensitivity is not high [3], and also the working temperature is imposed by the critical temperature of the superconductor and the use of a magnetic field is prohibited.

In the phonon echo method [4], the wave vector reversal, resulting from a parametric interaction between the elastic strain and the electric field, makes the acoustic wave travel back along the same path and the phase coherence is recovered on the emittingreceiving crystal face. This method is not competitive with the new one because it implies a piezoelectric detection and a large magnitude of the coefficient of the parametric interaction. Moreover it is restricted to piezoelectric materials [4] or necessitates a bond with a piezoelectric crystal.

The main advantage of the semiconductor avalanche bolometer is its independence of magnetic field and its rapid response time [6]. Its sensitivity is similar to that of the superconducting bolometer, therefore lower than that of our glass detector.

The possibility of using superconducting junctions (tunnel junctions [12] or Josephson junctions) as phonon detectors has been demonstrated. But in the case of low frequency phonons only Josephson junctions are effective [7]. Then it appears that for $10 \mathrm{GHz}$ ultrasonic waves they are less sensitive than piezoelectric detection and do not give an exponential decrease of the echo pattern.

5. Conclusion. - We have demonstrated the possibility of using a glass as an ultrasonic detector. The main advantage of this new method is the volume detection. Its sensitivity is higher than that of bolometric detection. It can operate in a broad range of temperatures and frequencies. In principle the glass can also be used to detect incoherent phonons (heat pulses).

\section{References}

[1] TUCKER, J. W. and RAMPTON, V. W., Microwave Ultrasonics in Solid State Physics (North Holland, Amsterdam) 1972.

[2] Jacobsen, E. H., J. Acoust. Soc. Am. 32 (1960) 949.

[3] Andrews Jr, J. M. and Strandberg, M. W. P., J. Appl. Phys. 38 (1967) 2660.

[4] Frénois, Ch., Joffrin, J. and Levelut, A., J. Physique 34 (1973) 747.

[5] CoHen, M. G. and Gordon, E. I., Bell Syst. Tech. J. 44 (1965) 693.

[6] Wigmore, J. K., Appl. Phys. Lett. 13 (1968) 73.

[7] Tredwell, T. J. and Jacobsen, E. H., Phys. Lett. 50A (1974) 281.
[8] HUnklinger, S. and ARNold, W. in Physical Acoustics, edited by R. N. Thurston and W. P. Mason (Academic Press, New York) 1976, Vol. 12, p. 155.

[9] Doussineau, P., Levelut, A. and Ta Thu-Thuy, J. Physique Lett. 38 (1977) L-37.

[10] Laermans, C., Arnold, W. and Hunklinger, S., J. Phys. C 10 (1977) L-161.

[11] Bachellerie, A., Doussineau, P. Levelut, A. and Ta ThuThuY, J. Physique 38 (1977) 69.

[12] Eisenmenger, W., Physical Acoustics (Academic Press) 1976, Vol. XII, p. 79. 